Esta publicación científica en formato digital es continuidad de la revista impresa ISSN-Versión Impresa 0798-1406 / ISSN-Versión on line 2542-3185Depósito legal pp $197402 Z$ U34

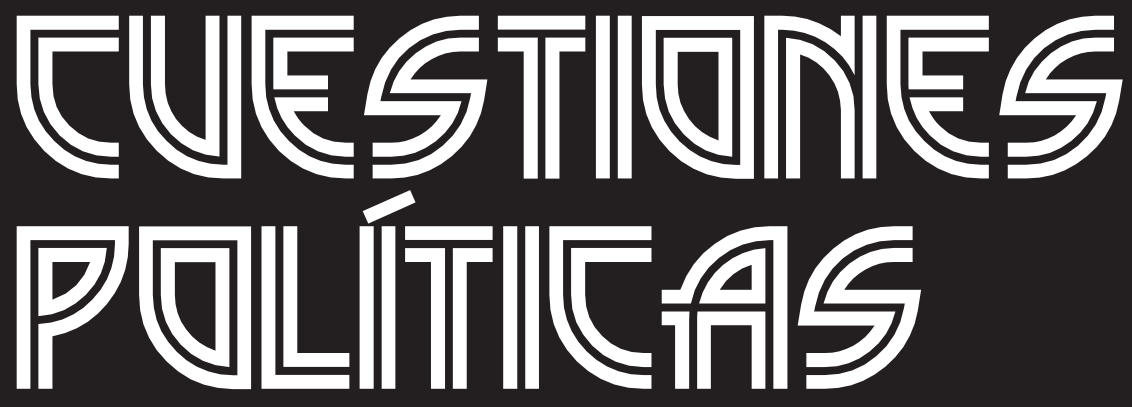

Instituto de Estudios Políticos y Derecho Público "Dr. Humberto J. La Roche" de la Facultad de Ciencias Jurídicas y Políticas de la Universidad del Zulia Maracaibo, Venezuela
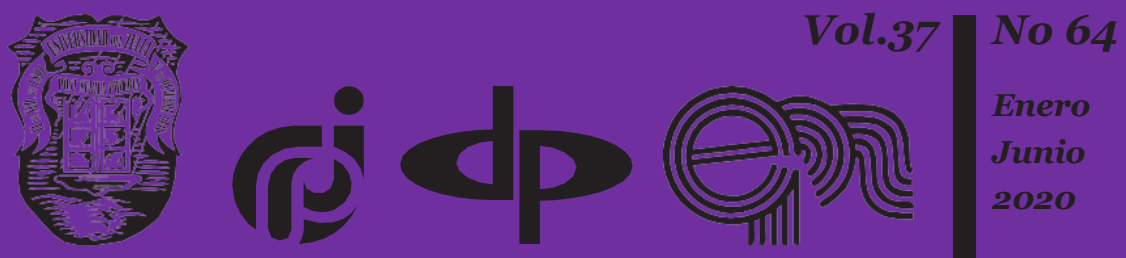


\title{
Balance de artículos de Derecho de origen ucraniano en la Revista Amazonia Investiga
}

\author{
Diego Felipe Arbeláez-Campillo * \\ Magda Julissa Rojas-Bahamón **
}

\section{Resumen}

La producción científica de artículos en el ámbito del Derecho ocupa un lugar destacado en las revistas arbitradas de alto impacto, bien sea por el volumen de artículos que se publican en cada número o por la trascendencia de un área del saber que se ocupa de la arquitectura del estado de Derecho, el funcionamiento de las instituciones democráticas, el goce y disfrute de los derechos fundamentales y la gestión del conflicto social, entre otros temas de interés. El objetivo general de esta investigación radica en desarrollar un balance bibliográfico sobre la producción de artículos de Derecho por parte de investigadores de Ucrania, publicados en la revista Amazonia Investiga en 2019, lo que subsume dar cuenta de: a) temas investigados, b) metodologías y técnicas de recolección de datos, c) fuentes empleadas y referentes teóricos y, d) aportes para el desarrollo de las ciencias jurídicas. En lo metodológico, se trata de una investigación próxima a la hermenéutica, ganada a interpretar el sentido profundo de 31 textos jurídicos indagados en el marco de la observación documental, a modo de técnica de recolección y organización de la información recabada. Entre las conclusiones más notorias destacan que, la producción científica en Derecho de los investigadores ucranianos está a tono con los problemas y tendencias actuales que identifican a la ciencia jurídica en el mundo de hoy.

Palabras clave: balance bibliográfico; producción científica en el ámbito del derecho; Ucrania; revista Amazonia Investiga; economía política.

* Magister en Educación. Universidad de la Amazonia, Colombia. Orcid: https:/orcid.org/oooo-ooo29041-9563. Email: dfaca@hotmail.com

** PhD. Educación y cultura ambiental. Profesora en la Institución Educativa Jorge Eliecer Gaitán, Universidad de la Amazonia Colombia. Email: mjulissa@gmail.com 


\title{
Balance of articles of Law of Ukrainian origin in Amazonia Investiga Journal
}

\begin{abstract}
The scientific production of articles in the field of Law occupies a prominent place in the arbitrated journals of high impact, either by the volume of articles published in each issue or by the transcendence of an area of knowledge that deals with architecture of the rule of law, the functioning of democratic institutions, the enjoyment and enjoyment of fundamental rights and the management of social conflict, among other topics of interest. The general objective of this research is to develop a bibliographic balance sheet on the production of Law articles by researchers from Ukraine, published in the Amazonia Investiga Journal in 2019, which subsumes to account for: a) investigated topics, b) methodologies and data collection techniques, c) sources used and theoretical references and, d) contributions to the development of legal science. In the methodological, it is a research close to hermeneutics, won to interpret the profound meaning of 31 legal texts investigated in the framework of documentary observation, as a technique for collecting and organizing the information collected. Among the most notorious conclusions are that, the scientific production in Law of Ukrainian researchers is in tune with the current problems and trends that identify legal science in the world today.
\end{abstract}

Keywords: bibliographic balance; scientific production in the field of law; Ukraine; Amazonia Investiga Journal; political economy.

\section{Introducción}

En las sociedades democráticas el derecho adquiere un rol fundamental, por un lado, sirve de marco normativo racional para la conducta de personas e instituciones, lo que genera certeza en cuanto a las decisiones y procedimientos que pueden esperarse para gestionar conflictos individuales y colectivos o para garantizar el goce y disfrute de los derechos positivos y subjetivos. Por el otro, el derecho es la garantía del mantenimiento de las instituciones y valores democráticos que postulan, desde los albores del programa filosófico de la modernidad y su ideología liberal, al individuo ciudadano como protagonista de la historia y persona humana dotada de una dignidad transcendental que los ordenamientos políticos y jurídicos deben garantizar y proteger en todo momento, con independencia de su forma y contenido.

Por estas circunstancias, la producción científica de artículos en el ámbito del derecho ocupa un lugar destacado en las revistas arbitradas de 
Diego Felipe Arbeláez-Campillo y Magda Julissa Rojas-Bahamón
Balance de artículos de Derecho de origen ucraniano en la Revista Amazonia Investiga.

alto impacto, bien sea por el volumen de artículos que se publican en cada número o por la importancia de un área del saber al mismo tiempo teórico y práctico, sin el cual, las sociedades humanas retornarían al estado de naturaleza donde prevalece la voluntad del más fuerte y estarían a merced del uso arbitrario del poder público para beneficio de autócratas y tiranos exclusivamente.

El objetivo general de esta investigación radica en desarrollar un balance bibliográfico sobre la producción de 31 artículos de Derecho de investigadores ucranianos, publicados en la revista Amazonia Investiga en 2019, órgano divulgativo independiente de carácter multidisciplinario y circulación bimensual, que publica resultados de investigación y reflexiones en los dominios de las humanidades, las ciencias sociales, la educación, tecnologías de la información y comunicación, cultura ambiental y salud, entre otros, tal como se evidencia en su portal web (https://www. amazoniainvestiga.info/index.php/amazonia).

La resolución del objetivo planteado implicó además dar cuenta de cuatro criterios concretos a saber: a) temas investigados, b) metodologías y técnicas de investigación, c) fuentes empleadas y referentes teóricos y, d) aportes para el desarrollo de las ciencias jurídicas. No obstante, cada trabajo revisado es particular y habló por sí mismo, lo que no significa que no existan tendencias comunes e intereses científicos compartidos en la comunidad de académicos de Odessa.

El artículo que hoy se presenta para contribuir con el desarrollo del debate y la reflexión sobre la producción científica en el ámbito del Derecho, generada por los académicos referidos, se desarrolló en cinco apartados autónomos pero interrelacionados. En el primero se da cuenta del alcance, límites y significados del problema de estudio y de cómo fue formulado por el equipo de investigación para operativizar el objetivo plateado. En el segundo, se muestra en líneas generales el proceso metodológico empleado y las tendencias epistemológicos que identifican nuestra concepción del conocimiento científico. En el tercer subtítulo, se efectúa el balance bibliográfico propiamente dicho con base a los cuatro criterios planteados anteriormente. En el cuarto apartado, se procedió a analizar y discutir los resultados obtenidos, para enunciar finalmente, los principales hallazgos de la investigación en las conclusiones. 


\section{El problema investigado}

El estudio que se presenta significa un balance bibliográfico que da cuenta de lo que se ha escrito en el ámbito del derecho y, de cómo se ha escrito, en un tiempo y órgano divulgativo determinado, por parte de una específica comunidad de investigadores. Se trató de elaborar una especie de estado del arte que, según se mire, pude servir para diversos propósitos de investigación, tales como: proporcionar una visión panorámica de la producción científica de los juristas ucranianos, indicar sus intereses temáticos, comparar la muestra seleccionada con lo que se produce sobre Derecho en otro contexto diferente o, determinar las falencias y contribuciones de los productos analizados. Todo dependerá de la creatividad y lectura crítica que nuestros amables lectores hagan en su momento de este insumo de investigación.

Como se sabe, muchas veces las fronteras que se conciben entre una ciencia o disciplina y otra, son muy tenues, por lo que pueden rebasarse con facilidad. Mucho más cuando se trata de investigaciones desarrollados bajo un enfoque holístico e interdisciplinario de las ciencias jurídicas, de ahí que el balance efectuado integró también trabajos desarrollados en criminología o en ciencias políticas que, no obstante, también poseen naturaleza jurídica si se leen de forma atenta y sosegada. En el cuadro que sigue se muestra la relación lógica que se dio entre el triángulo: objetivo general, criterios o unidades de análisis y la formulación del problema.

Objetivo general: desarrollar un balance bibliográfico sobre la producción de artículos de derecho de investigadores ucranianos, publicados en la revista Amazonia Investiga en 2019.

\begin{tabular}{|l|l|}
\hline $\begin{array}{l}\text { Criterios o unidades de } \\
\text { análisis: }\end{array}$ & Formulación del problema: \\
\hline a) temas investigados. & ¿Qué temas jurídicos se investigan? \\
\hline $\begin{array}{l}\text { b) metodologías y técnicas de } \\
\text { investigación. }\end{array}$ & $\begin{array}{l}\text { ¿Cuáles metodologías y técnicas de } \\
\text { investigación se emplean? }\end{array}$ \\
\hline $\begin{array}{l}\text { c) fuentes empleadas y referentes } \\
\text { teóricos. }\end{array}$ & $\begin{array}{l}\text { ¿Qué tipo fuentes se utilizan y con } \\
\text { qué referentes teóricos se } \\
\text { interpretan? }\end{array}$ \\
\hline $\begin{array}{l}\text { d) aportes para el desarrollo de las } \\
\text { ciencias jurídicas. }\end{array}$ & $\begin{array}{l}\text { ¿Qué aportes teóricos y/o } \\
\text { metodológicos se observan en los } \\
\text { artículos revisados? }\end{array}$ \\
\hline
\end{tabular}

Cuadro No 1. Sistematización de la investigación. Elaboración propia con base al objetivo general. 
Por lo demás, los resultados de esta investigación deben interpretarse como una muestra que configura una visión parcial y limitada de la producción científica en derecho que se genera en los académicos ucranianos, que podrá servir de insumo para futuras investigaciones más amplias y profundas en cuanto a la muestra de documentos procesados, el tipo y medio de publicación y al contexto temporal del estudio.

\section{Aspectos metodológicos y epistemológicos del estudio}

$\mathrm{Al}$ momento de leer las fuentes documentales recabadas en la selección bibliográfica, nos aproximamos al método hermenéutico que articula en una lectura crítica los textos en su contexto para acceder al "verdadero significado" de lo que los autores quieren comunicar en su obra de forma clara o solapada. Este tipo de lectura simultáneamente pueda dar cuenta de los intereses del investigador o investigadores, de sus sesgosideológicos e, inclusive, de su cosmovisión del mundo en el que les tocó vivir, así como de sus contradicciones; todo ello visualizando o imaginando en lo posible las características de su contexto existencial como fuerza simbólica que condiciona su producción académica.

Compartimos con (Moreno, 2008) la idea de que la hermenéutica entraña una interpretación comprensiva de realidades textuales y contextuales en la que se fusionan dos horizontes históricos: por un lado, la tradición en la que se sitúa el texto y su creador y, por el otro, al que pertenece el sujeto interpretante; muchas veces se trata de tradiciones muy distintas. Por consiguiente, todo acto exegético es en sí mismo dialógico por lo que remite a una compresión histórica, finita y limitada, nunca a la lectura final o correcta. La teoría hermenéutica clásica proporciona de igual modo tres niveles de lectura: significado textual, significado intertextual y sentido contextual, niveles que fueron de mucho valor para este trabajo.

De conformidad con la naturaleza del estudio se empleó la observación documental a modo de técnica para operativizar el método y para recolectar la información. Al decir de (Arias, 2006) la observación documental se expresa en un proceso sistemático de: “(...) búsqueda, recuperación, análisis, critica e interpretación de datos secundario, es decir, los obtenidos y registrados por otros investigadores en fuentes documentales: impresas, audiovisuales o electrónicas." Como en toda investigación, el propósito de este diseño es el aporte de nuevos conocimientos."

Los autores de este estudio comparten una concepción post-positivista de las ciencias sociales que admite la imposibilidad de traducir al lenguaje de lo cuantificable y medible, todos los fenómenos socioculturales y los actos 
interpretativos como condición para garantizar su cientificidad y justificar su validez. Concretamente, defendemos una postura epistemológica -holística y constructivista- que valora en igual de condiciones la dimensión objetiva y subjetiva de los saberes al tiempo que, supone en el conocimiento en general, una construcción intelectual que sirve de reflejo a la realidad y de modelo explicativo de sus fenómenos (Barrera, 2010). Para el constructivismo el sujeto conocedor no solo edifica las teorías que interpretan a la realidad social, sino que al mismo tiempo construye a la realidad misma desde su acción científica o transformadora, de ahí que saber y hacer son procesos interconectados.

\section{Estado del arte}

En el presente apartado se exponen de forma sucinta los aspectos más relevantes de los 31 artículos ubicados en el proceso de selección, realizado en función del objetivo planteado.

1. (Fedchyshyn, Ignatenko, \& Shvydka, 2019). Tema investigado: Diferencias económicas y legales en los patrones de uso de la tierra en Ucrania. Fuentes y referentes teóricos: La mayoría de las fuentes consultadas son secundarias, se usaron otros artículos arbitrados sobre el tema. No se evidencia el empleo de un modelo teórico en particular. Aportes al desarrolla de la ciencia jurídica: Se demuestra como el arrendamiento de tierras es la práctica o patrón de uso más extendido de las tierras agrícolas y cuáles son las funciones que cumple en el campo de Ucrania. Destacan sus funciones sociales, que van creando condiciones para el acceso a los recursos de la tierra de quienes no las poseen, al tiempo que propician el espíritu empresarial en los dominios de la agro- producción. Metodología: La base metodológica del estudio fue un complejo de métodos de conocimiento científico filosóficos (dialécticos, sistémicoestructurales) y legales especiales (formalmente lógicos, comparativoslegales, pronósticos y métodos de interpretación.

2. (Davydova, Orzikh, Samoylenko, Andronov, \& Pysarenko, 2019). Tema investigado: Consecuencias legales de la invalidez de una transacción realizada utilizando tecnología de la información. Fuentes y referentes teóricos: En líneas generales se citan artículos científicos. No se visualiza el uso de un modelo teórico en particular. Aportes al desarrollo de la ciencia jurídica: Con base a los resultados obtenidos se apuesta a la creación de un nuevo concepto de ley en la sociedad de la información con el desarrollo explícito de un 
mecanismo legal apropiado para la protección de los derechos de los participantes en estas transacciones en particular. Lo que demanda la introducción del concepto de la invalidez de las transacciones de TI y la visualización de sus implicaciones legales. Metodología: Se usaron métodos de análisis y síntesis para determinar la naturaleza y el propósito de una transacción no válida como objeto de relaciones legales civiles. Además, se utilizó el método formal y lógico para formular la definición de una transacción no válida

3. (Mudrak, Hloviuk, Murzanovska\& Voloshyna, 2019). Tema investigado: Condición fisiológica y psicológica de la víctima de un delito penal en Ucrania. Fuentes y referentes teóricos: Se recabo evidencia empírica concreta con la aplicación del cuestionario, al tiempo que se consultaron también fuentes secundarias. Se trabajó con el enfoque de consecuencias psicógenas, que se centra en los cambios en la condición mental y el comportamiento de las víctimas, como consecuencia de la ocurrencia del delito. Aportes al desarrollo de la ciencia jurídica: Los autores concluyen que la víctima debe ser reconocida como el sujeto de derecho a la asistencia legal gratuita (a expensas del estado), teniendo en cuenta en todo momento su condición psicoemocional y, en algunos casos, también la condición fisiológica, cognitiva y conductual, lo que incide en la incapacidad de proteger los derechos e intereses legítimos de la misma. Metodología: Se hizo uso del método del cuestionario para descubrir los problemas de los sujetos estudiados. La encuesta se dirigió a una muestra de 50 científicos en el área y 31 personas.

4. (Kharytonov, Kharytonova, Tolmachevska, Fasii \& Tkalych, 2019). Tema investigado: Seguridad de la información y medios de apoyo legal. Fuentes y referentes teóricos: En líneas generales se citaron fuentes secundarias de carácter académico. Aportes al desarrollo de la ciencia jurídica: El artículo aporta a modo de innovación heurística del tema, el concepto de higiene de la información. Los autores llegan a la conclusión de que es necesario introducir una regulación legal de las relaciones de información tanto a nivel internacional como nacional. Metodología: La base metodológica para el estudio fue un método dialéctico que permitió revisar los problemas en su desarrollo e interconexión. Se utilizaron métodos de análisis y síntesis para determinar la naturaleza de la seguridad de la información como objeto de las relaciones jurídicas- civiles.

5. (Tsybulska, Voronina, Fomichova, Tokareva \& Matiiko, 2019). Tema investigado: Influencia del derecho privado romano en los principios básicos de la sucesión singular en el derecho de herencia de Ucrania, Polonia y Lituania Fuentes y referentes teóricos: Comúnmente se utilizaron 
fuentes legales y artículos arbitrados que directa o indirectamente dan cuenta sobre el tema. No se visualiza un enfoque teórico en particular. Aportes al desarrollo de la ciencia jurídica: Los autores muestran como ciertos esquemas jurídicos provenientes de la tradición romana aún están presentes en las legislaciones modernas. Por lo demás, en la ley civil moderna de Ucrania, con la ayuda de un legado, el testador tiene la oportunidad de asignar al heredero la voluntad de ejecución de cualquier obligación de propiedad a favor de la persona, el legatario especificado por el testador. Polonia y Lituania también tienen una sucesión singular muy similar a la de Ucrania. Metodología: Los autores del artículo enuncian la utilización de enfoques dialécticos, sinérgicos, antropológicos, filosóficos, formal-lógicos, sistémicos, históricos, comparativos-legales, formaleslegales y otros métodos de investigación.

6. (Pyvovar, Detiuk, Mykolenko, Zapototska \& Lapka, 2019). Tema investigado: Apoyo Legal al Sistema de Control Estatal en el Proceso Educativo Gubernamental. Fuentes y referentes teóricos: Comúnmente se utilizaron fuentes legales, informes y artículos arbitrados que directa 0 indirectamente dan cuenta sobre el tema. Aportes al desarrollo de la ciencia jurídica: Los resultados de la investigación mostraron que el sistema de control estatal sobre el proceso educativo en Ucrania aún es muy débil. Los resultados del análisis de la legislación mostraron además que el control indirecto en este campo solo lo proporcionan organismos especiales para verificar la efectividad del uso de los fondos presupuestarios en el proceso de orden gubernamental educativo. $\mathrm{N}$ este contexto, hay violaciones sistemáticas a las leyes que rigen la materia y no se atiende la calidad educativa que es lo fundamental. Metodología. Se utilizaron métodos hermenéuticos, normativos y analíticos para averiguar el contenido de las fuentes de regulación legal de las órdenes gubernamentales educativas. Se afirma que los métodos de pronóstico, modelos legales y analogía de la ley contribuyeron al desarrollo de propuestas para mejorar la legislación actual.

7. (Kharytonov, Kharytonova, Kharytonova, Kolodin \& Tolmachevska, 2019). Tema investigado: Los derechos humanos como valor básico del concepto de derecho privado en la Europa moderna. Fuentes y referentes teóricos: La mayoría de las citas son de artículos arbitrado y textos especializados sobre el tema. Se evidencia un cierto manejo de la teoría dialéctica en el discurso. Aportes al desarrollo de la ciencia jurídica: Entre los aportes más significativos destaca la idea de que, la civilización occidental (europea) no abandona los valores "universales" tradicionales, aunque intenta en algunos casos determinar sus límites. En este sentido, se muestras ejemplos de censura bastante estricta, especialmente en el 
campo del cine, cuando las razones de la restricción fueron determinadas precisamente por consideraciones de protección de los valores universales, situación que en cierta forma resulta contradictoria. Por lo tanto, no hay motivos para percepciones excesivas sobre el "infinito" de los derechos humanos. Este límite se encuentra en los mismos límites del pensamiento lógico y racional que identifica a occidente. Metodología. Al decir de los autores en el proceso de investigación, se utilizaron métodos de investigación dialécticos, sinérgicos, sistémicos, históricos, legales comparativos y otros.

8. (Nekit, Ulianova \& Kolodin, 2019). Tema investigado: Sitio web como objeto de protección legal por la legislación de Ukrania. Fuentes y referentes teóricos: Entre las fuentes citas destacan artículos arbitrados, textos jurídicos, decisiones de tribunales sobre la materia y cuerpos normativos. No se aprecia el uso de un enfoque teórico en particular. Aportes al desarrollo de la ciencia jurídica: Se demuestra que el sitio web y el nombre de dominio son objetos independientes separados en el campo civil. Un nombre de dominio no es una parte integral de un sitio web y no se debe pasar de forma predeterminada al enajenar un sitio web. Conviene prestar especial atención a la protección del contenido del sitio web del plagio y la piratería, así como la responsabilidad por la información inexacta publicada en el sitio, para lo cual no se descarta el diseño de leyes más específicas que rijan la materia. Metodología: Según los autores la base metodológica para el estudio fue el método dialéctico que permitió revisar los problemas en su desarrollo e interconexión. Del mismo modo, se enuncia la utilización del método analítico, de síntesis ycomparativo.

9. (Adakhovska, Omelchuk, Gofman, Budurova \& Snizhanna, 2019). Tema investigado: Reglamentación legal de la herencia de bienes personales electrónicos. Fuentes y referentes teóricos: Se utilizan fuentes legalesy artículos arbitradas sobre eltema. No sevisualiza la adopción de un enfoque teórico en particular. Aportes al desarrollo de la ciencia jurídica: Al decir de los autores, los problemas de herencia de páginas y cuentas personales no están regulados por la ley civil vigente. Además, las normas legislativas que regulan relaciones de contenido similares no pueden aplicarse a las relaciones investigadas por varias razones, incluidos momentos éticos, posible violación de los derechos humanos y problemas tecnológicos. Por lo demás, las cuestiones de la herencia de los activos digitales requieren un estudio fundamental a nivel de la ciencia del derecho civil, con la posterior consolidación legislativa de los principios básicos de la regulación legal de estas relaciones. Metodología: En el proceso de investigación, se utilizaron métodos de investigación dialécticos, sinérgicos, sistémicos, históricos, legales comparativos y otros. 
10. (Safonchyk, Sirko \& Andronova, 2019). Tema investigado: Regulación de las relaciones matrimoniales por contrato. Fuentes y referentes teóricos: Se citaron fuentes secundarias en la modalidad de artículos científicos básicamente. No se observa apego a ningún enfoque teórico en particular dentro del capo de la ciencia jurídica. Aportes al desarrollo de la cienciajurídica: Los autores concluyeron queel contrato matrimonial es una herramienta destinada principalmente a fortalecer la institución familiar, permitiendo tener más en cuenta los intereses de cada cónyuge, reducir el número de disputas y conflictos entre ellos y, en el caso de divorcio y división de propiedad, gestiona los inconvenientes de forma civilizada. Metodología: Según los autores se combinaron diferentes metodologías, tales como: el método de investigación de sistemas, el método de derecho comparado, el método de análisis complejo y el método de modelado legal.

11. (Voloshyna V., Shylin, Cheremnova, Prytuliak, \& Stepanets, 2020). Tema investigado: Contratos empresariales de show: análisis jurídico civil de la legislación ucrania con experiencia de aplicación internacional Fuentes y referentes teóricos: Se citaron fuentes secundarias en la modalidad de artículos científicos básicamente. No se observa apego a ningún enfoque teórico en particular dentro del capo de la ciencia jurídica. Aportes al desarrollo de la ciencia jurídica: Los autores determinaron que las estructuras contractuales que rigen los espectáculos en Ucrania pueden resultar precarias al ser insuficientes para regular ciertas relaciones y aspectos que surgen en un show. Además, las actividades de las partes en el cumplimiento de sus obligaciones en virtud del contrato se caracterizan por un alto nivel de riesgo porque es muy difícil predecir el éxito de un proyecto en particular en el mundo del espectáculo. Metodología: En el estudio se utilizaron los siguientes métodos de investigación: análisis, síntesis, inducción y deducción.

12. (Kolodin, Kolodina \& Kaminskyi, 2019). Tema investigado: La protección del sufragio a través de recursos legales. Fuentes y referentes teóricos: Se citaron fuentes secundarias en la modalidad de artículos científicos básicamente. En lo teórico se plantean varias herramientas propias de las ciencias forenses. Aportes al desarrollo de la ciencia jurídica: Los autores destacaron las medidas más prometedoras para la protección de los derechos electorales de los ciudadanos y establecieron que existen dos formas principales de protección del sufragio: judicial y extrajudicial. Cuando se trata de defender la democracia y la voluntad ciudadana, se pueden combinar en una misma estrategia ambas formas de protección. Metodología: Según los autores los métodos específicos que se utilizarán en el estudio fueron: el legal comparativo, forense y formal. 
13. (Karakash, Riabokon, Popsuienko, Babych \& Zaveriukha, 2019). Tema investigado: Sucesión singular debido a la herencia en la ley romana antigua. Fuentes y referentes teóricos: La mayoría de las fuentes citadas son historiográficas. Aportes al desarrollo de la ciencia jurídica: A juicio de los autores, los romanos dividieron la sucesión en universal y singular ya que podría relacionarse con la propiedad como un todo y, como consecuencia, con todas sus partes constituyentes, o solo ciertas partes de la propiedad según el interés del autor del testamento. Metodología: Según los autores se trata de una metodología dialéctica e histórica.

14. (Bakaianova, Svyda, Demenchuk, Dzhaburiya \& Fomina, 2019). Tema investigado: Abogacía de Ucrania bajo reforma constitucional. Fuentes y referentes teóricos: Se citaron fuentes secundarias en la modalidad de artículos científicos básicamente. No se evidencia el uso de ningún enfoque teórico en particular. Aportes al desarrollo de la ciencia jurídica: Se proponen cambios en los códigos procesales para garantizar en todo momento el equilibrio óptimo de la libertad de elección. En el entendido que, dichos cambios no compensan las actividades irresponsables de ningún representante, con o sin educación legal. De cualquier modo, el abogado debe seguir siendo el representante exclusivo, único y "externo" que brinda asistencia legal profesional. Metodología: los autores señalan que usaron métodos científicos generales: análisis, síntesis, inducción y deducción en la investigación.

15. (Khyzhniak, Mandrychenko, Pidgorodynska, Khylchenko \& Marieiev, 2019). Tema investigado: Problemas de criminalización de las violaciones de los derechos de los pacientes: experiencia internacional y situación actual en Ucrania. Fuentes y referentes teóricos: Se citaron fuentes secundarias en la modalidad de artículos científicos básicamente. No se evidencia el uso de ningún enfoque teórico en particular. Aportes al desarrollo de la ciencia jurídica: Los autores afirman la posibilidad de crear un banco de órganos de donantes genéticamente idénticos y extender la vida de una persona en particular, pero sin desconocer el peligro de cambiar la naturaleza de los humanos, lo que conducirá a la muerte completa de Homo sapiens moderno. Debido a la falta de regulación, los derechos reproductivos en Ucrania permanecen prácticamente desprotegidos. Por lo tanto, es necesario incluir un artículo separado en el Código Penal de Ucrania, que imponga castigos por las violaciones de los derechos humanos reproductivos. Metodología: Según los autores se emplearon los métodos de análisis, síntesis, inducción, deducción, entre otros. 
16. (Drobush, Olkina, Vodopian \& But, 2019). Tema investigado: Garantía constitucional del derecho a la protección judicial en disputas relacionadas con la invalidez de un testamento como base para la adquisición de derechos de propiedad en Ucrania. Fuentes y referentes teóricos: Se citan fuentes hemerográficas y cuerpos legales. No se observa la adopción de un enfoque teórico en particular. Aportes al desarrollo de la ciencia jurídica: Según los resultados del estudio, se concluyó que las normas de la Constitución junto al derecho civil y la legislación procesal civil de Ucrania sirven como sustento para una generalización de la jurisprudencia en disputas sobre la invalidez de un testamento, como base para adquirir derechos de propiedad en Ucrania. Metodología: Se argumentó haber usado métodos generales de investigación científica y, el método dialectico en particular.

17. (Goncharenko, Spasova, \& Kalitenko, 2019). Tema investigado: Protección de los derechos civiles y los intereses legales en Ucrania. El artículo analiza cuestiones legales generales, teóricas y prácticas sobre la protección de los derechos e intereses civiles, la esencia del interés legal como un objeto específico de protección, determinando las formas de protección del derecho civil. Fuentes y referentes teóricos: Se abordan disposiciones del instituto para la protección de los derechos e intereses establecidos por el Código Civil de la URSS y el Código Civil de Ucrania, así como las normas de Derecho civil de algunos países europeos. Aportes al desarrollo de la ciencia jurídica: Se distinguen los campos específicos del interés legal, el interés legítimo y el derecho subjetivo en relación con la forma jurisdiccional de protección por parte del Estado. Metodología: sistema de métodos de modelado filosófico general (dialéctico, histórico), científico general (método de análisis sistemático, estructural-funcional, retrospectivo-comparativo), y métodos de investigación especiales (lógicos formales, comparativos, estadísticos).

18. (Volkova, Prytuliak, Tsal-Tsalko, Yanitska, \& Poliuk, 2019). Tema investigado: Características generales del reclamo en los países del derecho anglosajón y continental. Fuentes y referentes teóricos: Análisis de disposiciones legales de Estados Unidos de América, Alemania y Reino Unido en materia de demandas y acciones colectivas. Aportes al desarrollo de la ciencia jurídica: Los autores caracterizan la naturaleza legal de la demanda, definiendo el concepto de la demanda en los actos normativos-legales de los países indicados. Metodología: la base de la metodología del estudio fueron los métodos generales científicos y especiales de conocimiento de los fenómenos legales.

19. (Basay, Hryniuk, \& Kovalchuk, 2019). Tema investigado: Normas de prueba en el procedimiento penal de los Estados Unidos de América y Ucrania mediante una investigación comparativa. Fuentes y referentes teóricos: La 
investigación parte de los preceptos del Código Modelo de Procedimiento Penal de EE. UU. y el Código de Procedimiento Penal de Ucrania. Aportes al desarrollo de la ciencia jurídica: Se analizan las normas de prueba en el procedimiento penal del derecho consuetudinario y los sistemas jurídicos continentales (en el ejemplo de los Estados Unidos y Ucrania). Los autores indican que la comprensión de las normas de prueba está estrechamente vinculada a la suficiencia de las pruebas en el momento de la adopción de la decisión procesal pertinente, que se establece por el resultado de su evaluación. Metodología: El fundamento metodológico del trabajo es un sistema de métodos filosóficos, científicos generales y específicos de la investigación científica: método legal comparativo, método sistemático, método funcional y método legal formal.

20. (Reznichenko, Herasymchuk, \& Shemonayev, 2019). Tema investigado: Características generales y tipos de derechos de propiedad sobre la propiedad de otra persona (iura in re aliena) en la legislación civil de Ucrania. Fuentes y referentes teóricos: En líneas generales se citan artículos arbitrados, textos historiográficos y fuentes legales. Se percibe el uso de conceptos y categorías de análisis típicos del discurso histórico. Aportes al desarrollo de la ciencia jurídica: Se exponen las limitaciones estructurales de la legislación existente que regula el desarrollo de los derechos de propiedad extranjera en el derecho civil moderno de Ucrania y, al mismo tiempo se exhibe la necesidad de enmendar la legislación existente que necesita reforma, ya que aún no puede proporcionar las condiciones legales para el desarrollo de los derechos de propiedad extranjera en la práctica de las relaciones concretas. Metodología: Se emplea el método histórico-comparativo.

21. (Reznichenko, Yanitska, \& Apalkova, 2019). Tema investigado: Indemnización por daños causados a la vida y la salud de los participantes en el conflicto armado en Ucrania. Fuentes y referentes teóricos: Ley de Ucrania sobre protección social y legal del personal militar y familiares, así como el Código Civil de Ucrania, la Constitución y demás leyes de carácter social. Aportes al desarrollo de la ciencia jurídica: Se llega a la conclusión de que el término "ganancias perdidas" solo se puede aplicar al personal militar que sirve en el reclutamiento militar, ya que para ellos se tomarán como base las ganancias que un ciudadano tenía antes de ser reclutado para el servicio militar. Las pérdidas de propiedad causadas al personal militar en virtud del contrato se calculan sobre la base de sus beneficios en efectivo y otros ingresos (no relacionados con el desempeño de los deberes militares). Metodología: Se utilizaron métodos científicos generales y especiales en el proceso de investigación. La base metodológica para el estudio fue un método dialéctico que permitió revisar los problemas en su desarrollo e interconexión. Se utilizaron métodos de análisis y síntesis para determinar los detalles de la compensación por daños causados a la vida y la salud de los participantes en un conflicto armado. Se utilizó un 
método comparativo para revelar las diferencias entre la compensación del daño a la propiedad y el daño moral.

22. (Fedorov, Chipko, \& Tishchenko, 2019). Tema investigado: determinar el lugar del derecho ucraniano entre otros sistemas de derecho en el mundo. Fuentes y referentes teóricos: El artículo se fundamenta en los aportes identificados en artículos científicos y publicaciones bibliográficas sobre el tema de la relación del derecho ucraniano con otros sistemas jurídicos del mundo. Aportes al desarrollo de la ciencia jurídica: Los autores concluyen que el sistema legal ucraniano necesita mejoras y una mayor integración en el sistema legal romanoalemán. Al mismo tiempo, algunas de las generaciones pasadas de abogados y académicos nacionales merecen ser repensados en la era moderna. Metodología: derecho comparado, análisis, síntesis, inducción, deducción,

23. (Davydova, Bernaz-Lukavetska, \& Fedenko, 2019). Tema investigado: Aspectos separados de los derechos de imagen que se utilizan en hologramas: experiencia mundial y problemas de la regulación legal Ucrania. Fuentes y referentes teóricos: Se parte del análisis del Código Civil de Ucrania y la revisión de fuentes especializadas como artículos científicos y material bibliográfico. Aportes al desarrollo de la ciencia jurídica: El estudio reveló el área más popular de uso de los derechos de imagen: el holograma. Se han abierto y analizado las posibilidades de elaboración de la regulación legislativa de las deficiencias legales en materia de protección y restauración de los derechos de imagen. Se dan respuestas a los detalles de la posibilidad de heredar y transferir derechos de imagen a terceros. Metodología: Métodos (histórico, comparativo), clasificación, generalización y abstracción.

24. (Hloviuk, Hryniuk, \& Kovalchuk, 2019). Tema investigado: Desafíos actuales para involucrar expertos en procesos penales por delitos económicos en Ucrania, con el propósito de sistematizar la especificidad de los motivos y el patrón de participación de un experto en procedimientos penales con respecto a delitos económicos. Fuentes y referentes teóricos: Se analizan las leyes de Ucrania en materia penal y a su vez se orienta la investigación mediante los aportes encontrados en artículos científicos y fuentes bibliográficas. Aportes al desarrollo de la ciencia jurídica: los autores recomiendan que en el Código de Procedimiento Penal de Ucrania se indique un precepto, obligando a las partes a tramitar la asignación de un experto que debe ser contratado, o una autoridad experta que debe llevar a cabo el examen; también el juez, para fundamentar su decisión debe apoyarse en un experto que realice el examen. Metodología: método sistemático de análisis funcional, histórico y legal, métodos regulatorios formales y lógicos. 
25. (Baitaliuk, Matviichuk, \& Moskovchuk, 2019). Tema investigado: Contrato de alquiler bajo la legislación de Ucrania, con el propósito de estudiar algunos aspectos del nuevo instituto contractual del derecho civil ucraniano: el Instituto de Alquiler. Fuentes y referentes teóricos: Análisis basado en el Código Civil de Ucrania y aportes teóricos de artículos científicos y fuentes bibliográficas. Aportes al desarrollo de la ciencia jurídica: Los autores llegaron a la conclusión de que la práctica de aplicar la construcción legal del contrato de alquiler, que se formó durante los 15 años del Código Civil, demuestra que el Instituto de Alquiler no está privado de ciertos problemas, lo que influye en la aplicación de la ley. Por lo tanto, siempre que se eliminen las lagunas legales y las deficiencias de cumplimiento, los participantes en la rotación civil utilizarán con mayor frecuencia el contrato de alquiler. Metodología: Método de análisis y síntesis, método dialéctico.

26. (Bakaianova N. , Svyda, Demenchuk, Dzhaburiya, \& Fomina, 2019). Tema investigado: Abogacía de Ucrania bajo reforma constitucional, mediante el análisis de los proyectos de ley sobre la abolición del monopolio del abogado, las opiniones de los organismos supranacionales sobre este tema. Fuentes y referentes teóricos: Constitución de Ucrania, Proyecto de ley sobre colegio de abogados de Ucrania, aportes de artículos científicos. Aportes al desarrollo de la ciencia jurídica: Los autores concluyeron que un abogado debe seguir siendo el representante exclusivo, único y "externo" que brinda asistencia legal profesional. Metodología: Se utilizaron métodos generales de investigación científica y jurídica en la redacción de la investigación.

27. (Karakash, Riabokon, Popsuienko, Babych,, \& Zaveriukha, 2019). Tema investigado: Sucesión singular debido a la herencia en la ley romana antigua, por medio del análisis de la herencia (universal o singular) por legatum y fideicommissum. Fuentes y referentes teóricos: Análisis de la tradición jurídica de Roma y revisión de artículos científicos. Aportes al desarrollo de la ciencia jurídica: los romanos dividieron la sucesión en universal y singular, ya que podría relacionarse con la propiedad como un todo, y como consecuencia - a todos sus constituyentes partes, o solo ciertas partes de la propiedad. Metodología: El método dialéctico como método general de conocimiento científico y método histórico permitió estudiar la herencia por legatum y fideicommissum; método de análisis del sistema, así como los métodos estructural y lógico formal del sistema; método comparativo-legal ayuda a explorar la experiencia de la antigua Roma de regular las relaciones de herencia; el método legal formal muestra las normas legales de la antigua legislación de Roma que regula los problemas de herencia. 
28. (Kolodin, Kolodina, \& Kaminskyi, 2019). Tema investigado: La protección del sufragio a través de recursos legales. Fuentes y referentes teóricos: Constitución de Ucrania y aportes sobre el tema en artículos científicos. Aportes al desarrollo de la ciencia jurídica: los autores destacaron las medidas más prometedoras para la protección de los derechos electorales de los ciudadanos y establecieron que existen dos formas principales de protección del sufragio: judicial y extrajudicial. Metodología: métodos científicos generales de análisis y síntesis, métodos dialécticos y lógicos. Los métodos específicos que se utilizarán en el estudio serán: legal comparativo, forense y formal.

29. (Nekit, Shershenkova, \& Voloshyna, 2019). Tema investigado: estudio de los detalles de la institución de propiedad fiduciaria en la legislación de Ucrania. Fuentes y referentes teóricos: Código Civil de Ucrania, disposiciones gubernamentales y aportes de artículos científicos. Aportes al desarrollo de la ciencia jurídica. La situación actual en la legislación ucraniana no nos permite sacar conclusiones inequívocas sobre si la propiedad fiduciaria seguirá calificándose como un tipo de derecho de propiedad o si ocupará su lugar en el sistema de derechos reales (aparte de la propiedad). Parece que la respuesta a esta pregunta será dada por la práctica, que eventualmente hará sus propios ajustes. Metodología: métodos de análisis y síntesis, métodos de inducción y deducción.

30. (Lahutina, Osadchyi, Zakalenko, Kozachuk, \& Bolshakova, 2019). Tema investigado: El concepto moderno de justicia administrativa en Ucrania. Fuentes y referentes teóricos: Constitución de Ucrania y resultados de artículos científicos relacionados con el tema de investigación. Aportes al desarrollo de la ciencia jurídica. La justicia administrativa es una manifestación de la autoridad pública: la justicia. La forma de implementación de este tipo de actividad, que refleja sus características esenciales, es el procedimiento administrativo (proceso administrativo). Metodología: método de comparación, métodos de análisis y síntesis, métodos de inducción y deducción, método formallegal.

31. (Svyda, Kovalchuk, Torbas, Melnychuk, \& Kytaika, 2019). Tema investigado: El problema de la regulación legal del derecho de una persona a un juicio justo, la necesidad de una definición legislativa del derecho de una persona a un juicio justo en el contexto de los derechos humanos. Fuentes y referentes teóricos: Constitución de Ucrania, acuerdos internacionales en materia de Derechos 
Humananos, aportes sobre el tema en artículoscientíficos. Aportes al desarrollo de la ciencia jurídica. El contenido del concepto de "juicio justo" de cubrir en tribunales los requisitos para la igualdad de acceso a la justicia, la publicidad de los casos, para garantizar el derecho del acusado, el cual debe ser informado en detalle sobre la naturaleza. y los motivos de los cargos en su contra, dándole el tiempo y la oportunidad suficientes para preparar su defensa, defenderse personalmente o elegir un abogado defensor para esa defensa, incluso de forma gratuita. Metodología: métodos de análisis y síntesis.

\section{Análisis y discusión de resultados}

La producción de artículos en el campo del Derecho por parte de investigadores ucranianos, publicados en la Revista Amazonia Investiga, durante el año 2019, puede interpretarse de la siguiente manera:

\subsection{Temas investigados}

Los temas planteados por los autores se insertan en los campos del Derecho civil, Derecho penal, Derecho mercantil, Derechos Humanos y Derecho comparado. En este sentido, destacan los estudios sobre aspectos de la realidad socio-política de Ucrania, donde se busca dar respuesta a la protección de los Derechos Humanos en el marco de los recientes conflictos que ha experimentado esta nación. También sobresalen los temas asociados con el marco legal en materia de tecnologías de la información y de la comunicación. Finalmente, los autores se interesan por el estudio del ámbito penal, así como el análisis de la propiedad y la herencia. De esta manera, la producción científica en Derecho de los investigadores ucranianos está a tono con los problemas y tendencias actuales que identifican a las Ciencias Jurídicas en el mundo de hoy (De Miguel Asensio, 2003).

\subsection{Metodologías y técnicas de investigación}

En general, los artículos publicados se limitan a presentar una síntesis de la metodología, para lo cual se recurre a la identificación de métodos generales de: análisis, síntesis, dialécticos y comparativos. Los autores 
no se detienen a explicar las técnicas empleadas en sus respectivas investigaciones, y de manera excepcional uno de los artículos (Mudrak, Hloviuk, Murzanovska, \& Voloshyna, 2019) plantea la aplicación de una encuesta a una determinada muestra. Puede decirse que, en su conjunto, los artículos responden a investigaciones documentales de nivel descriptivo, cuyo propósito apunta a la comprensión de los temas antes mencionados. De acuerdo con (Rojas Cairampoma, 2015, pág. 7) este tipo de investigaciones. "Exhibe el conocimiento de la realidad tal como se presenta en una situación de espacio y de tiempo dado. Aquí se observa y se registra, o se pregunta y se registra. Describe el fenómeno sin introducir modificaciones: tal cual. Las preguntas de rigor son: ¿Qué es?, ¿Cómo es?, ¿Dónde está?, ¿Cuándo ocurre?, ¿Cuantos individuos o casos se observan?, ¿Cuáles se observan?”.

\subsection{Fuentes empleadas y referentes teóricos}

Dada la naturaleza jurídica de las investigaciones, y considerando la procedencia geográfica de éstas y los temas tratados, se entiende la constante recurrencia al Código de Derecho Civil y la Constitución de Ucrania. Ambos instrumentos son interpretados con base a criterios jurídicos, que son reforzados mediante los aportes teóricos y conceptuales de autores de artículos científicos que son citados en cada uno de los trabajos. De esta manera se da curso a la argumentación jurídica, experiencia que está presente en todos los quehaceres de los juristas. De acuerdo con Manuel Atienza (Viloria Ochoa, 2016, págs. 34-35) existen varias modalidades de la argumentación, todas ellas presentes en los artículos identificados:

El autor plantea diversas concepciones de la argumentación, afirmando que se trata de un quehacer tan complejo, que no existe un enfoque único y suficiente desde el cual se la realice. Por ello, partiendo de un concepto general, distingue entre la argumentación formal, material y pragmática. La interpretación formal: (aspecto fundamental de la lógica deductiva estándar), considera la argumentación como una actividad compuesta de una serie de enunciados independientemente de su verdad, de la corrección de sus premisas o la verdad de sus conclusiones. La interpretación material: En ella lo esencial no es la forma de los argumentos, sino su contenido material, esto es las normas o hechos en tanto ser o deber ser. La concepción pragmática: hace alusión a un tipo de actividad que busca lograr la interacción del intérprete para el logro de la persuasión o el acuerdo con un auditorio, respetando ciertas reglas. 


\subsection{Aportes al desarrollo de las Ciencias Jurídicas}

Los resultados y conclusiones de los artículos examinados, ofrecen insumos para la comprensión de la realidad jurídica y sociopolítica en Ucrania. Hay en un grueso de los artículos identificados la incursión de sus autores en la hermenéutica jurídica, como experiencia alternativa al positivismo, para lograr "la comprensión de textos jurídicos (...) el sentido original de los mismos, en búsqueda del Derecho y del juicio correctos, teniendo en cuenta el momento actual. Por medio de dicha labor se procura una decisión o pronunciamiento esencialmente creativo, complementario y perfeccionador del Derecho" (Ossa, 2002, pág. 41). A partir de este ángulo epistemológico, se amplía la comprensión de temas sensibles como los Derechos Humanos, aspectos socioculturales (entre ellos la comunicación) y el desarrollo del Derecho penal ucraniano de los últimos años. Cabe destacar que el interés por estos tópicos y la forma como son abordados por parte de los articulistas ucranianos, coincide con la experiencia de sus pares en otras latitudes (Alvarado Villa, 2017), (Sánchez Bernal, 2012), (De Miguel Asensio, 2003).

\section{Conclusiones}

Los temas de reflexión de los articulistas ucranianos de la Revista Amazonia Investiga apuntan a la comprensión de dos ámbitos que son del interés de las Ciencias Jurídicas: por un lado, el contexto sociopolítico contemporáneo de Ucrania y las respuestas que el Estado ha dado al orden social que ha ido emergiendo, razón por la cual el Código Civil ha servido de instrumento para regular las relaciones de la ciudadanía y el propio Estado con la propiedad, la herencia, los derechos humanos y los procesos penales; de otro lado, se constata el interés de los autores por reflexionar acerca de las repercusiones de la globalización ( (De Miguel Asensio, 2003) en procesos que forman parte de las relaciones transfronterizas, entre estos: las comunicaciones y la transmisión de información, el comercio y los contratos empresariales, donde el Estado ucraniano no escapa a la pérdida del control, evidenciada en todos los Estados nacionales, sobre estas expresiones características de la globalización. 


\section{Referencias Bibliográficas}

ADAKHOVSKA, Nadiia; OMELCHUK, Oleksandr; GOFMAN, Oleksandr; BUDUROVA, Hanna; SNIZHANNA, Alina. 2019. "Legal regulation of the inheritance of personal electronic assets" En: Amazonia Investiga. Disponible en línea. En: https://amazoniainvestiga.info/index.php/ amazonia/article/viEw/108. Fecha de consulta: 09/01/2020.

ALVARADO VILLA, Numa Enrique. 2017. "Concepto de denuncia de la carta de la Organización de los Estados Americanos por el Estado venezolano" En: Revista de la Universidad del Zulia. Disponible en línea. En: https://www.produccioncientificaluz.org/index.php/rluz/article/ VIEW/29874/30854. Fecha de consulta: 09/01/2020.

ARIAS, Fidias G. 2006. El Proyecto de Investigación Introducción a la metodológica científica. Editorial Episteme. Caracas, Venezuela.

BAITALIUK, Dariia; MATVIICHUK, Alina; MOSKOVCHUK, Dmytro. 2019. "Rent Contract under the Legislation of Ukraine" En: Revista Amazonia Investiga. Disponible en línea. En: https://amazoniainvestiga.info/ index.PHP/AMAZONIA/ARTICLE/VIEW/910. Fecha de consulta: 09/09/2019.

BAKAIANOVA, Nana; SVYDA, Oleksii; DEMENCHUK, Maryna; Dzhaburiya, Olena; FOMINA, Oksana. 2019. "Advocacy of Ukraine under constitutional reform” En: Amazonia Investiga. Disponible en línea. En: https://amazoniainvestiga.info/index.php/amazonia/article/view/805. Fecha de consulta: 09/10/2019.

BARRERA, Marcos Fidel. 2010. Modelos epistémologicos en investigación y educación. Sypal Quirón Ediciones. Caracas, Venezuela.

BASAY, Viktor; HRYNIUK, Volodymyr; KOVALCHUK, Serhii. 2019. "Standards of proof in the criminal procedure of the United States of America and Ukraine: A comparative research" En: Revista Amazonia Investiga. Disponible en línea. En: https://www.amazoniainvestiga.info/index. PHP/AMAZONIA/ARTICLE/VIEW/801. Fecha de consulta: 15/01/2020.

DAVYDOVA, Iryna; BERNAZ-LUKAVETSKA, Olena; FEDENKO, Yelyzaveta. 2019. "Separate aspects of image rights using in holograms: world experience and problems of Ukrainian legal regulation" En: Revista Amazonia Investiga. Disponible en línea. En: https://amazoniainvestiga. info/index.php/amazonia/article/view/869. Fecha de consulta: 11/26/2019. 
Diego Felipe Arbeláez-Campillo y Magda Julissa Rojas-Bahamón

DAVYDOVA, Iryna; ORZIKH, Yurii; SAMOYLENKO, Georgiy; ANDRONOV, Ihor; PYSARENKO, Maryna. 2019. "Legal Consequences of the Invalidity of a Transaction made by Using Information Technology: Problems of Theory and Practice” En: Amazonia Investiga. Disponible en línea. En: https://www.amazoniainvestiga.info/index.php/amazonia/article/ VIEW/276/253. Fecha de consulta: 05/06/2019.

DE MIGUEL ASENSIO, Pedro Alberto. 2003. Algunas tendencias jurídicas de la globalización. In Los nuevos escenarios internacionales y europeos del Derecho y la seguridad. BOE-AEPDIRI. Madrid, España.

DROBUSH, Iryna; OLKINA, Olena; VODOPIAN, Tetiana; BUT, Illia. 2019. "Constitutional guarantee of the right to judicial protection in disputesconcerning the invalidity of a will as grounds for the acquisition of propertyrights in Ukraine" En: Amazonia Investiga. Disponible en línea. En: https://www.amazoniainvestiga.info/index.php/amazonia/ ARTICLE/VIEW/831. Fecha de consulta: 05/08/2019.

FEDCHYSHYN, Dmytro; IGNATENKO, Iryna; SHVYDKA, Viktoriia. 2019. "Economic and legal differences in patterns of land use in Ukraine" En: Amazonia Investiga. Disponible en línea. En: https://amazoniainvestiga. info/index.PHP/AMAZONIA/ARTICLE/VIEW/286. Fecha de consulta: 05/08/2019.

FEDOROV, Valentyn; CHIPKO, Nataliia; TISHCHENKO, Yuliia. 2019. "Place of Ukrainian law among other legal systems of the world" En: Revista Amazonia Investiga. Disponible en línea. En: https://amazoniainvestiga. info/index.php/amazonia/article/view/846. Fecha de consulta: 08/11/2019.

GONCHARENKO, Vlada; SPASOVA, Ekaterina; KALITENKO, Oksana. 2019. "Protection of civil rights and legal interests in Ukraine" En: Revista Amazonia Investiga. Disponible en línea. En: https://www. amazoniainvestiga.info/index.PHP/AMAZONIA/ARTICLE/VIEW/37/18. Fecha de consulta: 08/11/2019.

HLOVIUK, Iryna; HRYNIUK, Volodymyr; KOVALCHUK, Serhii. 2019. "Modern Challenges to Engagement an Expert in Criminal Proceedings on Economic Crimes in Ukraine" En: Revista Amazonia Investiga. Disponible en línea. En: https://amazoniainvestiga.info/index.php/ amazonia/article/vIEW/881. Fecha de consulta: 11/11/2019.

KARAKASH, Illya; RIABOKON, Ievgen; POPSUIENKO, Liudmyla; BABYCH, Iryna; ZAVERIUKHA, Maryna. 2019. "Singular succession due to inheritance in ancient Roman law" En: Amazonia Investiga. Disponible en línea. En: https://amazoniainvestiga.info/index.php/amazonia/ ARTICLE/VIEW/802. Fecha de consulta: 11/12/2019. 
KHARYTONOV, Evgen; KHARYTONOVA, Olena; KHARYTONOVA, Tetiana; KOLODIN, Denis; TOLMACHEVSKA, Yuliia. 2019. "Human rights as the basic value of the concept of privatelaw in modern Europe" En: Amazonia Investiga. Disponible en línea. En: https://amazoniainvestiga.info/ index.PHP/AMAZONIA/ARTICLE/VIEW/177. Fecha de consulta: 11/12/2019.

KHARYTONOV, Evgen; KHARYTONOVA, Olena; TOLMACHEVSKA, Yuliia; FASII, Bondan; TKALYCH, Maxym. 2019. "Information security and means of its legal support” En: Amazonia Investiga. Disponible en línea. En: https://www.amazoniainvestiga.info/index.php/amazonia/article/ VIEW/227. Fecha de consulta: 08/11/2019.

KHYZHNIAK, Yevhen; MANDRYCHENKO, Zhanna; PIDGORODYNSKA, Anastasiia; KHYLCHENKO, Anastasiia; MARIEIEV, Vasyl. 2019. "Problems of criminalization of violations of patients' rights: international experience and current situation in Ukraine" En: Amazonia Investiga. Disponible en línea. En: https://amazoniainvestiga.info/index.php/ amazonia/article/vIEW/827. Fecha de consulta: 08/11/2019.

KOLODIN, Denis; KOLODINA, Anna; KAMINSKYI, Petro. 2019. "The protection of suffrage through legal remedies" En: Amazonia Investiga. Disponible en línea. En: https://www.amazoniainvestiga.info/index. php/amazonia/article/view/534. Fecha de consulta: 11/12/2019.

LAHUTINA, Iryna; OSADCHYI, Anatolii; ZAKALENKO, Olena; KOZACHUK, Diana; BOLSHAKOVA, Olga. 2019. "The modern concept of Ukrainian administrative justice” En: Amazonia Investiga. Disponible en línea. En: https://amazoniainvestiga.info/index.PHP/AMAZONIA/ARTICLE/VIEW/925. Fecha de consulta: 08/11/2019.

MORENO, Alejandro. 2008. El aro y la trama. Episteme, modernidad y pueblo. Convivium Press. Miami, EUA.

MUDRAK, Inna; HLOVIUK, Iryna; MURZANOVSKA, Alina; VOLOSHYNA, Vladlena. 2019. "Physiological and psychological condition of victim of criminal offense in Ukraine" En: Amazonia Investiga. Disponible en línea. En: https://www.amazoniainvestiga.info/index.php/amazonia/ ARTICLE/VIEW/193. Fecha de consulta: 11/12/2019.

NEKIT, Kateryna; SHERSHENKOVA, Viktoria; VOLOSHYNA, Svitlana. 2019. "Fiduciary ownership in ukrainian legislation" En: Amazonia Investiga. Disponible en línea. En: https://amazoniainvestiga.info/index.php/ amazonia/article/view/944. Fecha de consulta: 11/23/2019.

NEKIT, Kateryna; ULIANOVA, Halyna; KOLODIN, Denis. 2019. "Website as an object of legal protection by Ukrainian legislation” En: Amazonia Investiga. /Vol. 8 Núm. 21: Julio-agosto, pp. 222- 230. 
OSSA, Mario. 2002. "Nuevas tendencias del derecho" En: Opinión Jurídica. Disponible en línea. En: https://revistas.udem.edu.co/index.php/ OPINION/ARTICLE/VIEW/1295. Fecha de consulta: 11/23/2019.

PYVOVAR, Yuriy; DETIUK, Andriy; MYKOLENKO, Oleksandr; ZAPOTOTSKA, Olena; LAPKA, Oksana. 2019. "Legal Support Of The System Of State Control In The Process Of Educational Governmental Order" En: Amazonia Investiga. Disponible en línea. En: https://amazoniainvestiga. info/index.php/amazonia/article/view/76. Fecha de consulta: $11 / 23 / 2019$.

REZNICHENKO, H; YANITSKA, I; APALKOVA, I. 2019. "Compensation for damage caused to the life and health of participants in the armed conflict in Ukraine” En: Revista Amazonia Investiga. Disponible en línea. En: https://amazoniainvestiga.info/index.php/amazonia/article/view/828. Fecha de consulta: 11/28/2019.

REZNICHENKO, S; HERASYMCHUK, L; SHEMONAYEV, V. 2019. "General characteristics and types of proprietary rights on another's property (iura in re aliena) in civil legislation of Ukraine” En: Amazonia Investiga. Disponible en línea. En: https://www.amazoniainvestiga.info/index. php/amazonia/article/view/785. Fecha de consulta: 11/28/2019.

ROJAS CAIRAMPOMA, Marcelo. 2015. "Tipos de Investigación científica: Una simplificación dela complicada incoherente nomenclatura y clasificación" En: REDVET. Revista Electrónica de Veterinaria. Disponible en línea. En: https://www.redalyc.org/pdf/636/63638739004.pdf. Fecha de consulta: 11/28/2019.

SAFONCHYK, Oksana; SIRKO, Rostyslav; ANDRONOVA, Viktoriia. 2019. "Regulation of marriage relations by contract" En: Amazonia Investiga. Disponible en línea. En: https://amazoniainvestiga.info/index.php/ amazonia/article/vIEW/115. Fecha de consulta: 11/28/2019.

SALMANOVA, Olena; POPOVICH, Evgen; NIKITIN, Anatoliy; TESLIUK, Irina. 2020. "Administrative and Legal Principles of the Digitization of Public Relations" En: Amazonia Investiga. Disponible en línea. En: https:// amazoniainvestiga.info/index.PHP/AMAZONIA/ARTICLE/VIEW/1088. Fecha de consulta: 11/28/2019.

SÁNCHEZ BERNAL, Javier. 2012. "Responsabilidad penal de las personas jurídicas” En: CT, Universidad del Salamanca, pp.121-156.

SVYDA, Tetiana; KOVALCHUK, Inna; TORBAS, Oleksandr; MELNYCHUK, Yuliia; KYTAIKA, Olga. 2019. "The formation and current tendencies of international human rights protection concerning the right of a person 
to a fair trial, and their impact on Ukraine" En: Amazonia Investiga. Disponible en línea. En: https://amazoniainvestiga.info/index.php/ amazonia/article/view/939. Fecha de consulta: 11/28/2019.

TSYBULSKA, Olha; VORONINA, Nadiia; FOMICHOVA, Natalia; TOKAREVA, Vira; MATIIKO, Mykola. 2019. "Influence of Roman private law on the basic principles of singular succession in the inheritance law of Ukraine, Poland and Lithuania" En: Amazonia Investiga. Disponible en línea. En: https://amazoniainvestiga.info/index.php/amazonia/article/view/74. Fecha de consulta: 11/28/2019.

VILORIA OCHOA, José Gregorio. 2016. "La argumentación jurídica y sus teorías: una aproximación a la propuesta de Manuel Atienza" En: Revista de la Universidad del Zulia. Disponible en línea. En: https:// produccioncientificaluz.org/index.php/rluz/article/vIEW/29901. Fecha de consulta: 12/26/2019.

VOLKOVA, Nataliia; PRYTULIAK, Valerii; TSAL-TSALKO, Yuliia; YANITSKA, Inna; POLIUK, Yuliia. 2019. "General characteristics of the claim in the countries of the anglo-saxon and continental law" En: Revista Amazonia Investiga. Disponible en línea. En: https://www.amazoniainvestiga.info/ index.php/amazonia/article/view/535. Fecha de consulta: 12/26/2019.

VOLOSHYNA, Vladlena; SHYLIN, Denys; CHEREMNOVA, Antonina; PRYTULIAK, Valerii; STEPANETS, Yurii. 2019. "Show business contracts: civil legal analysis of ukraine legislation with international application experience" En: Amazonia Investiga. Disponible en línea. En: https://www.amazoniainvestiga.info/index.php/amazonia/article/ view/38. Fecha de consulta: 11/28/2019. 


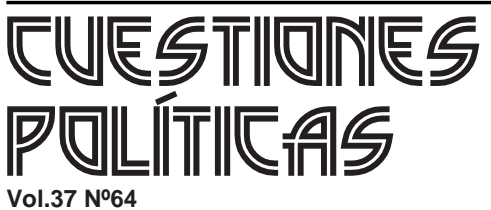

Esta revista fue editada en formato digital y publicada en junio de 2020, por el Fondo Editorial Serbiluz, Universidad del Zulia. Maracaibo-Venezuela

www.luz.edu.ve

www.serbi.luz.edu.ve

www.produccioncientificaluz.org 